\title{
BOUNDARY NON-CROSSING PROBABILITIES OF GAUSSIAN PROCESSES: SHARP BOUNDS AND ASYMPTOTICS
}

\author{
ENKELEJD HASHORVA, YULIYA MISHURA, AND GEORGIY SHEVCHENKO
}

\begin{abstract}
We study boundary non-crossing probabilities
\end{abstract}

$$
P_{f, u}:=\mathrm{P}\left(\forall t \in \mathbb{T} X_{t}+f(t) \leq u(t)\right)
$$

for continuous centered Gaussian process $X$ indexed by some arbitrary compact separable metric space $\mathbb{T}$. We obtain both upper and lower bounds for $P_{f, u}$. The bounds are matching in the sense that they lead to precise logarithmic asymptotics for the large-drift case $P_{y f, u}, y \rightarrow+\infty$, which are two-term approximations (up to $o(y)$ ). The asymptotics are formulated in terms of the solution $\tilde{f}$ to the constrained optimization problem

$$
\|h\|_{\mathbb{H}_{X}} \rightarrow \min , \quad h \in \mathbb{H}_{X}, h \geq f
$$

in the reproducing kernel Hilbert space $\mathbb{H}_{X}$ of $X$. Several applications of the results are further presented.

\section{INTRODUCTION}

In this article we are interested in boundary non-crossing probabilities

$$
P_{f, u}:=\mathrm{P}\left(\forall t \in \mathbb{T} X_{t}+f(t) \leq u(t)\right) .
$$

Here $X$ is a continuous centered Gaussian process defined on a compact separable metric space $\mathbb{T}, u: \mathbb{T} \rightarrow \mathbb{R}$ (boundary) and $f: \mathbb{T} \rightarrow \mathbb{R}$ (trend) are some deterministic functions. The continuity assumption is motivated by the observation that in order for the probability to be well defined, the corresponding event has to be generated by values of $X$ on some countable subset of $\mathbb{T}$. Two most natural situations when this happens are the case of countable $\mathbb{T}$ (which we will study elsewhere) and the case of a continuous process defined on a separable metric space, studied here. We further restrict ourselves to the more tractable case of compact $\mathbb{T}$ (and we also show how the case of locally compact $\mathbb{T}$ can be reduced to it). Sufficient conditions for continuity of Gaussian process are given in e.g. [23. Chapter 10].

Explicit formulas for $P_{f, u}$ are known only for very special $X$ and particular $u, f$ with most prominent example $X$ being a Wiener process and $u, f$ being piece-wise linear functions, see e.g., [22, 26, 28]. In the absence of explicit formulas, several authors have obtained upper and lower bounds for the non-crossing probabilities of Gaussian processes with trend and/or their asymptotic behavior. We list just few references on such question: Wiener process was considered in [4, 11, 18]; Brownian bridge, in [3, 5, 7]; Brownian pillow and Brownian sheet in [19, 8]; additive Wiener field, in [20]; fractional Brownian motion, in [21]; for closely related investigations, see [24, 27].

2010 Mathematics Subject Classification. 60G15; 60G70; 60F10.

Key words and phrases. Gaussian process, boundary non-crossing probability, large deviations, reproducing kernel Hilbert space, Cameron-Martin theorem, constrained quadratic optimization problem, compact metric space.

${ }^{1}$ Further we impose stronger assumptions on the drift than on the boundary and also consider the asymptotics of $P_{y f, u}$ when $y \rightarrow+\infty$, and for this reason we do not write the probability in question as $\mathrm{P}\left(\forall t \in \mathbb{T} X_{t} \leq g(t)\right)$ with $g(t)=u(t)-f(t)$. 
In the case where $\mathbb{T}=[0, T]$ the boundary non-crossing probabilities $P_{f, u}$ are related to survival probabilities

$$
\mathrm{P}\left(\forall t \in \mathbb{T} X_{t}+f(t)<u(t)\right)=\mathrm{P}\left(\tau_{u}>T\right),
$$

where $\tau_{u}=\inf \left\{t \geq 0: X_{t}+f(t) \geq u(t)\right\}$ is the hitting time of a moving boundary $u$. Such probabilities (typically their asymptotic behavior as $T \rightarrow \infty$ ) are studied in the now very active topic of persistence probabilities. We refer to [2] for a comprehensive review of the topic.

Under the continuity assumption, the process $X$ can be regarded as a centered Gaussian element in the separable Banach space (equipped with supremum norm)

$$
C_{0}\left(\mathbb{T} ; \mathbb{T}_{0}\right):=\left\{g \in C(\mathbb{T}): \forall t \in \mathbb{T}_{0} \quad g(t)=0\right\}
$$

of continuous functions vanishing on the zero set $\mathbb{T}_{0}:=\left\{t \in \mathbb{T}: X_{t}=0\right.$ a.s. $\}$ of $X$. The zero set of the process is emphasized since the crucial role in asymptotic results is played by the injectivity of the covariance operator, which is defined on the dual space. In case of $C_{0}\left(\mathbb{T} ; \mathbb{T}_{0}\right)$ its dual is the space $M\left(\mathbb{T}_{1}\right)$ of signed finite measures on $\mathbb{T}_{1}=\mathbb{T} \backslash \mathbb{T}_{0}$. If the process were considered as an element of $C(\mathbb{T})$, the dual would be $M(\mathbb{T})$, and the kernel of the covariance operator will contain the measures supported by $\mathbb{T}_{0}$. So such a setting is chosen to allow for the greatest generality (and note that $\mathbb{T}_{0}$ may be empty).

Our approach to getting bounds for $P_{f, u}$ is based on the change of measure with the help of Cameron-Martin theorem. For this reason we assume that $P_{0, u} \in(0,1)$ and the drift $f$ belongs to the Cameron-Martin space (or reproducing kernel Hilbert space, RKHS) $\mathbb{H}_{X}$ of $X$. The latter is defined in terms of the covariance function

$$
R(t, s)=\mathrm{E}\left[X_{t} X_{s}\right], \quad t, s \in \mathbb{T}
$$

as the completion of the space spanned by $R(t, \cdot)$ with respect to the scalar product defined as a linear extension of

$$
(R(t, \cdot), R(s, \cdot))_{\mathbb{H}_{X}}=R(t, s) .
$$

The Cameron-Martin space can be also described in terms of the covariance operator, defined by

$$
\langle\mathcal{R} \mu, \nu\rangle=\mathrm{E}[\langle X, \mu\rangle\langle X, \nu\rangle], \mu, \nu \in M\left(\mathbb{T}_{1}\right),
$$

where $\langle\cdot, \cdot\rangle$ denotes the duality pairing.

A general lower bound for $P_{f, u}$ follows from [1, Proposition 1.6]. For any $f \in \mathbb{H}_{X}$, let $\tilde{f}$ be the metric projection of zero to the closed convex set $C_{f}:=\left\{h \in \mathbb{H}_{X}, h \geq f\right\}$. Then, applying [1, Proposition 1.6] with $S=C_{f}-f$ and the drift $f-\tilde{f}$, we get

$$
P_{f, u} \geq P_{f-\tilde{f}, u} \exp \left\{-\frac{1}{2}\|\tilde{f}\|_{\mathbb{H}_{X}}^{2}-\|\tilde{f}\|_{\mathbb{H}_{X}} \sqrt{-2 \log P_{f-\tilde{f}, u}}\right\} .
$$

We note in passing that comparable lower bounds to (11) follow also by [22, Theorem 1.1'] or [23, Theorem 7.3]. From the above, if further $P_{0, u} \in(0,1)$, then

$$
\log P_{y f, u} \geq-\frac{y^{2}}{2}\|\tilde{f}\|_{\mathbb{H}_{X}}^{2}+O(y), \quad y \rightarrow+\infty
$$

The main (and a hard) problem is the derivation of an accurate upper bound for $P_{y f, u}$ (which matches (2)) valid for all large $y$. One approach goes through the general large deviation principle for Gaussian measures, it is explained in detail in Subsection 2.4.

In this contribution we show that a sharp upper bound for $P_{f, u}$ can be determined if there exists a non-negative finite measure $\tilde{\gamma} \in M\left(\mathbb{T}_{1}\right)$ such that $\tilde{f}=\mathcal{R} \tilde{\gamma} \geq f$ and 
$(f-\tilde{f}, \tilde{f})_{\mathbb{H}_{X}} \geq 0$. In this case we establish in Theorem 2.3 the following upper bound:

where

$$
P_{f, u} \leq P_{0, u} \exp \left\{-\frac{1}{2}\|\tilde{f}\|_{\mathbb{H}_{X}}^{2}+\Theta(\tilde{\gamma}, u)\right\}
$$

$$
\Theta(\tilde{\gamma}, u)=\int_{\mathbb{T}_{1}} u(t) \tilde{\gamma}(d t)
$$

and a similar lower bound. Under the additional assumption that the operator $\mathcal{R}$ is injective and some special assumption on $\mathbb{H}_{X}$, we identify $\tilde{f}$ with the aforementioned projection and prove that

$$
\log P_{y f, u}=-\frac{y^{2}}{2}\|\tilde{f}\|_{\mathbb{H}_{X}}^{2}+y \Theta(\tilde{\gamma}, u)+o(y), \quad y \rightarrow+\infty
$$

which implies an equality in (2) and further refines the asymptotics.

In the special case where $X$ is a standard Wiener process, $\tilde{f}$ is the least non-decreasing concave majorant of $f$, and the above asymptotics agrees with the known results for Brownian motion, see e.g. [4].

The paper is organized as follows. The main results of the article are displayed in Section 2, which starts with a brief introduction to Gaussian processes. In Subsection 2.1, we establish both upper and lower bounds for $P_{f, u}$. The obtained results are then used to derive logarithmic asymptotics of non-crossing probabilities in Subsection 2.2. Subsection 2.3 shows how the results can be extended to the case of a locally compact parameter space. In Subsection 2.4, we show how an asymptotic upper bound matching (16) can be derived using the general large deviation principle for Gaussian measures. In Section 3, we illustrate the findings of Section 2 considering several important Gaussian processes. Section A contains some auxiliary results.

\section{MAin RESUlts}

Throughout the paper, $(\Omega, \mathcal{F}, \mathrm{P})$ is a complete probability space carrying all objects under consideration.

As in the Introduction, $X=\left\{X_{t}, t \in \mathbb{T}\right\}$ is a continuous centered real-valued Gaussian process defined on some compact separable metric space $(\mathbb{T}, \tau)$, with covariance function $R(t, s)$, which (thanks to continuity of $X$ ) is continuous in both $t$ and $s$. The zero set $\mathbb{T}_{0}=\left\{t \in \mathbb{T}: X_{t}=0\right.$ a.s. $\}$ of $X$ is closed and can be given in terms of the covariance function:

$$
\mathbb{T}_{0}=\{t \in \mathbb{T}: R(t, t)=0\}=\{t \in \mathbb{T}: \forall s \in \mathbb{T} R(t, s)=0\}
$$

Recall that the process $X$ is a Gaussian element in the separable Banach space $C_{0}\left(\mathbb{T} ; \mathbb{T}_{0}\right)$ of functions vanishing on $\mathbb{T}_{0}$, whose dual is $M\left(\mathbb{T}_{1}\right)$. Hereafter $\langle\cdot, \cdot\rangle$ shall denote the duality pairing between $C_{0}\left(\mathbb{T} ; \mathbb{T}_{0}\right)$ and $M\left(\mathbb{T}_{1}\right)$ i.e.

$$
\langle x, \mu\rangle=\int_{\mathbb{T}} x(t) \mu(d t), x \in C_{0}\left(\mathbb{T} ; \mathbb{T}_{0}\right), \mu \in M\left(\mathbb{T}_{1}\right)
$$

as well as between other spaces and their duals. We slightly abuse notation here since $\mu$ is not defined on $\mathbb{T}_{0}$; there is no danger since $x(t)=0$ for $t \in \mathbb{T}_{0}$.

The covariance operator $\mathcal{R}: M\left(\mathbb{T}_{1}\right) \rightarrow C_{0}\left(\mathbb{T} ; \mathbb{T}_{0}\right)$ corresponding to $X$ can be equivalently defined by

or

$$
\mathcal{R} \mu(t)=\int_{\mathbb{T}} R(t, s) \mu(d s), \mu \in M\left(\mathbb{T}_{1}\right)
$$

$$
\langle\mathcal{R} \mu, \nu\rangle=\mathrm{E}[\langle X, \mu\rangle\langle X, \nu\rangle]=\int_{\mathbb{T}} \int_{\mathbb{T}} R(t, s) \mu(d s) \nu(d t), \mu, \nu \in M\left(\mathbb{T}_{1}\right)
$$


Since $R$ is a non-negative definite function, (44) defines an inner product on the quotient of $M\left(\mathbb{T}_{1}\right)$ modulo ker $\mathcal{R}$. The completion of the latter with respect to this inner product is the Hilbert space of so-called measurable linear functionals, which will be denoted by $\mathcal{H}_{X}$, and the corresponding inner product will be denoted by $(\cdot, \cdot)_{\mathcal{H}_{X}}$. Moreover, thanks to (44), the operator $\mathcal{R}$ can be extended to $\mathcal{H}_{X}$ by continuity so that

$$
\left(\mu_{1}, \mu_{2}\right)_{\mathcal{H}_{X}}=\left\langle\mathcal{R} \mu_{1}, \mu_{2}\right\rangle, \mu_{1} \in \mathcal{H}_{X}, \mu_{2} \in M\left(\mathbb{T}_{1}\right) .
$$

Again, by continuity, the above duality pairing can be extended to $\mu_{1}, \mu_{2} \in \mathcal{H}_{X}$. Similarly, by (44), $\langle X, \cdot\rangle$ can be extended to an isometry between $\mathcal{H}_{X}$ and some subspace of $L^{2}(\Omega)$.

It is also worth noting that for any $\mu \in \mathcal{H}_{X}$, the random variable $\langle X, \mu\rangle$, being a mean square limit of centered Gaussian random variables, is a centered Gaussian random variable with variance $\mathrm{E}\left[\langle X, \mu\rangle^{2}\right]=\|\mu\|_{\mathcal{H}_{X}}^{2}$.

Remark 2.1. We slightly abuse rigor here, because the space $\mathcal{H}_{X}$ is a completion of the quotient $M\left(\mathbb{T}_{1}\right) /$ ker $\mathcal{R}$, not a completion of $M\left(\mathbb{T}_{1}\right)$. For example, the book [23] goes through $I^{*}: M\left(\mathbb{T}_{1}\right) \rightarrow M\left(\mathbb{T}_{1}\right) /$ ker $\mathcal{R}$. However, we decided to keep this slightly ambiguous notation for the sake of clarity and simplicity and in view of the fact that the main results of this article are formulated for the case where $\mathcal{R}$ is injective.

Further, $\mathcal{R}$ defines an isometry between $\mathcal{H}_{X}$ and its image $\mathbb{H}_{X}=\mathcal{R} \mathcal{H}_{X}$ equipped with the inner product

$$
\left(\mathcal{R} \mu_{1}, \mathcal{R} \mu_{2}\right)_{\mathbb{H}_{X}}=\left(\mu_{1}, \mu_{2}\right)_{\mathcal{H}_{X}}
$$

Defining for $t \in \mathbb{T}$ the Dirac measure $\delta_{t}$ by $\left\langle x, \delta_{t}\right\rangle=x(t), x \in C_{0}\left(\mathbb{T} ; \mathbb{T}_{0}\right)$, we have

$$
(R(t, \cdot), R(s, \cdot))_{\mathbb{H}_{X}}=\left(\mathcal{R} \delta_{t}, \mathcal{R} \delta_{s}\right)_{\mathbb{H}_{X}}=\left(\delta_{t}, \delta_{s}\right)_{\mathcal{H}_{X}}=R(t, s)
$$

for all $t, s \in \mathbb{T}$, so the space $\mathbb{H}_{X}$ is indeed the reproducing kernel Hilbert space (RKHS) of $X$, since it is unique with respect to the covariance reproducing property (5).

We present below the classical Cameron-Martin theorem for $X$, see [23, Theorem 5.1]. The formulation in [23] is given in terms of push-forward measures induced by $X$ and $X+f$ and is slightly different from the one given below, but it is easily seen to be equivalent.

Lemma 2.2 (Cameron-Martin theorem). If $f=\mathcal{R} \mu \in \mathbb{H}_{X}$, then the distribution of $X+f$ with respect to $\mathrm{P}$ is the same as that of $X$ with respect to the measure $\mathrm{P}^{f}$ with

$$
\frac{d \mathrm{P}^{f}}{d \mathrm{P}}=\mathcal{E}_{X}(\mu):=\exp \left\{\langle X, \mu\rangle-\frac{1}{2}\|\mu\|_{\mathcal{H}_{X}}^{2}\right\}=\exp \left\{\langle X, \mu\rangle-\frac{1}{2}\|f\|_{\mathbb{H}_{X}}^{2}\right\} .
$$

2.1. Bounds for non-crossing probabilities. In this section, we study the boundary non-crossing probability

$$
P_{f, u}:=\mathrm{P}\left(\forall t \in \mathbb{T} \quad X_{t}+f(t) \leq u(t)\right) .
$$

Here $f \in \mathbb{H}_{X}$ and $u: \mathbb{T} \rightarrow \mathbb{R}$ is a lower semicontinuous function such that $P_{0, u}>0$. The assumption of lower semicontinuity of $u$ does not harm the generality. Indeed, in view of the continuity of $X$ and $f$, for any bounded function $u: \mathbb{T} \rightarrow \mathbb{R}$ we have

$$
\left\{\forall t \in \mathbb{T} \quad X_{t}+f(t) \leq u(t)\right\}=\left\{\forall t \in \mathbb{T} \quad X_{t}+f(t) \leq u_{*}(t)\right\},
$$

where $u_{*}$ is the lower semicontinuous envelope of $u$.

Further we derive lower and upper bounds for $P_{f, u}$ for any trend $f \in \mathbb{H}_{X}$. Denote by $M^{+}\left(\mathbb{T}_{1}\right)$ the set of finite non-negative measures on $\mathbb{T}_{1}$ and recall that $\Theta(\tilde{\gamma}, u)=$ $\int_{\mathbb{T}_{1}} u(t) \tilde{\gamma}(d t)$.

Theorem 2.3. Let $f \in \mathbb{H}_{X}$ and suppose that $\tilde{f}=\mathcal{R} \tilde{\gamma}$ with $\tilde{\gamma}$ satisfying condition

$$
\text { (G1) } \tilde{\gamma} \in M^{+}\left(\mathbb{T}_{1}\right) \text {. }
$$

1. If the condition 


$$
(\mathrm{G} 2)\langle f-\tilde{f}, \tilde{\gamma}\rangle \geq 0
$$

is satisfied, then

$$
P_{f, u} \leq P_{f-\tilde{f}, u} \exp \left\{-\frac{1}{2}\|\tilde{f}\|_{\mathbb{H}_{X}}^{2}+\Theta(\tilde{\gamma}, u)\right\} .
$$

2. Let $u_{-}: \mathbb{T} \rightarrow \mathbb{R}$ be a continuous function such that $u_{-}(t)<u(t)$ for all $t \in \mathbb{T}$. If further

$$
P_{0, u, u_{-}}:=\mathrm{P}\left(\forall t \in \mathbb{T} \quad u_{-}(t) \leq X_{t} \leq u(t)\right)>0
$$

and condition

$$
\text { (G3) } \tilde{f} \geq f \text {, i.e. } \tilde{f}(t) \geq f(t) \text { for all } t \in \mathbb{T}
$$

holds, then

$$
P_{f, u} \geq P_{0, u, u_{-}} \exp \left\{-\frac{1}{2}\|\tilde{f}\|_{\mathbb{H}_{X}}^{2}+\Theta\left(\tilde{\gamma}, u_{-}\right)\right\} .
$$

Proof. 1. Using Lemma 2.2 we have

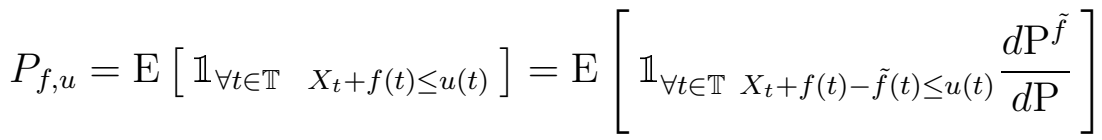

$$
\begin{aligned}
& =\mathrm{E}\left[\mathbb{1}_{\forall t \in \mathbb{T} X_{t}+f(t)-\tilde{f}(t) \leq u(t)} \exp \left\{-\frac{1}{2}\|\tilde{f}\|_{\mathbb{H}_{X}}^{2}+\langle X, \tilde{\gamma}\rangle\right\}\right] \text {. }
\end{aligned}
$$

Note that

(9) $\langle X, \tilde{\gamma}\rangle \leq\langle X+f-\tilde{f}, \tilde{\gamma}\rangle=\int_{\mathbb{T}_{1}}\left(X_{t}+f(t)-\tilde{f}(t)\right) \tilde{\gamma}(d t) \leq \int_{\mathbb{T}_{1}} u(t) \tilde{\gamma}(d t)=\Theta(\tilde{\gamma}, u)$

on $\left\{\forall t \in \mathbb{T} X_{t}+f(t)-\tilde{f}(t) \leq u(t)\right\}$ thanks to (G1) and (G2). Thus, we get

$$
\begin{gathered}
P_{f, u} \leq \mathrm{E}\left[\mathbb{1}_{\forall t \in \mathbb{T}} \quad X_{t}+f-\tilde{f} \leq u(t)\right. \\
\left.=P_{f-\tilde{f}, u} \exp \left\{-\frac{1}{2}\|\tilde{f}\|_{\mathbb{H}_{X}}^{2}+\Theta(\tilde{\gamma}, u)\right\}\right] \\
\left.\|\tilde{f}\|_{\mathbb{H}_{X}}^{2}+\Theta(\tilde{\gamma}, u)\right\}
\end{gathered}
$$

establishing the claim.

2. From assumption (G3), namely $\tilde{f} \geq f$, we obtain similarly to (8)

$$
\begin{aligned}
& P_{f, u}=\mathrm{E}\left[\mathbb{1}_{\forall t \in \mathbb{T}} \quad X_{t}+f(t) \leq u(t)\right] \geq \mathrm{E}\left[\mathbb{1}_{\forall t \in \mathbb{T}} X_{t}+\tilde{f}(t) \leq u(t)\right] \\
& \quad=\mathrm{E}\left[\mathbb{1}_{\forall t \in \mathbb{T}} X_{t} \leq u(t)\right. \\
& \geq \exp \left\{-\frac{1}{2}\|\tilde{f}\|_{\mathbb{H}_{X}}^{2}+\langle X, \tilde{\gamma}\rangle\right] \\
& \geq\left[\mathbb{1}_{\forall t \in \mathbb{T}} u_{-}(t) \leq X_{t} \leq u(t)\right. \\
& \exp \left\{-\frac{1}{2}\|\tilde{f}\|_{\mathbb{H}_{X}}^{2}+\langle X, \tilde{\gamma}\rangle\right] .
\end{aligned}
$$

Also, similarly to (9), we obtain

$$
\langle X, \tilde{\gamma}\rangle \geq \Theta\left(\tilde{\gamma}, u_{-}\right)
$$

on $\left\{\forall t \in \mathbb{T} u_{-}(t) \leq X_{t} \leq u(t)\right\}$, whence

$$
P_{f, u} \geq P_{0, u, u_{-}} \exp \left\{-\frac{1}{2}\|\tilde{f}\|_{\mathbb{H}_{X}}^{2}+\Theta\left(\tilde{\gamma}, u_{-}\right)\right\} .
$$


Remark 2.4. From (G1) and (G3) it follows that $\langle f-\tilde{f}, \tilde{\gamma}\rangle \leq 0$, so (G2) holds as an equality whenever (G1)-(G3) are satisfied simultaneously for some $\tilde{\gamma}$ and $\tilde{f}$ (not necessarily equal to $\mathcal{R} \tilde{\gamma})$. Moreover, in this case $\tilde{\gamma}$ and $\tilde{f}-f$ must be "orthogonal" in the sense that $\tilde{\gamma}$ is supported by the set $\left\{t \in \mathbb{T}_{1}: \tilde{f}(t)-f(t)=0\right\}$.

Now we turn to the question of identification of $\tilde{f}$ and $\tilde{\gamma}$ satisfying (G1)-(G3). To this end, for any $f \in \mathbb{H}_{X}$, consider the following minimization problem:

$$
\text { minimize }\|h\|_{\mathbb{H}_{X}} \text { for all } h \in \mathbb{H}_{X}, h \geq f
$$

here the comparison is understood, as usual, in the pointwise sense, i.e. $h \geq f$ means $h(t) \geq f(t)$ for all $t \in \mathbb{T}$.

Lemma 2.5. The set $C_{f}:=\left\{h \in \mathbb{H}_{X} \mid \forall t \in \mathbb{T} h(t) \geq f(t)\right\}$ is a closed set in $\mathcal{H}_{X}$.

Proof. Since $\mathbb{H}_{X}$ consists of continuous functions, we can consider the identity operator $\operatorname{id}_{\mathbb{H}_{X}}$ as acting from $\mathbb{H}_{X}$ to $C_{0}\left(\mathbb{T} ; \mathbb{T}_{0}\right)$. It is obviously closed, so by the continuous graph theorem it is continuous. Consequently, the set $C_{f}$, which is closed in $C_{0}\left(\mathbb{T}^{\prime} ; \mathbb{T}_{0}\right)$, is also closed in $\mathbb{H}_{X}$.

Since the set $C_{f}$ is convex and closed in $\mathbb{H}_{X}$, then by [17, Chapter 1], there exists a unique element $\tilde{f}$ solving the minimization problem (10). Moreover, the following proposition holds.

Proposition 2.6. The solution to the minimization problem (10) satisfies

$$
(f-\tilde{f}, \tilde{f})_{\mathbb{H}_{X}}=0 .
$$

Proof. Since $\tilde{f}$ is a metric projection of 0 to the set $C_{f}$, the solution $\tilde{f}$ is characterized by the well-known variational inequality (see e.g., [12, Lemma] or [25, Lemma 2.2])

$$
\|\tilde{f}\|_{\mathbb{H}_{X}}^{2} \leq(\tilde{f}, h)_{\mathbb{H}_{X}} \quad \forall h \in C_{f} .
$$

Plugging $h=f$ to (12), we get $(f-\tilde{f}, \tilde{f})_{\mathbb{H}_{X}} \geq 0$; plugging $h=2 \tilde{f}-f$, we obtain $(f-\tilde{f}, \tilde{f})_{\mathbb{H}_{X}} \leq 0$, establishing the claim.

In other words, any $\tilde{\gamma}$ such that $\mathcal{R} \tilde{\gamma}=\tilde{f}$ satisfies (G2) and (G3). To ensure the validity of (G1), we need an additional assumption.

(P) If $g \in \mathcal{H}_{X}$ is such that $\langle f, g\rangle \geq 0$ for all $f \in \mathbb{H}_{X}^{+}:=\left\{f \in \mathbb{H}_{X} \mid f \geq 0\right\}$, then $g \in M^{+}\left(\mathbb{T}_{1}\right)$.

Proposition 2.7. Under the assumption $(P)$, the solution $\tilde{f}=\mathcal{R} \tilde{\gamma}$ to the minimization problem (10) satisfies (G1)-(G3).

Proof. If $k \in \mathbb{H}_{X}^{+}$, then $\tilde{f}+k \in C_{f}$, hence by (12)

$$
\|\tilde{f}\|_{\mathbb{H}_{X}}^{2} \leq(\tilde{f}, \tilde{f}+k)_{\mathbb{H}_{X}}=\|\tilde{f}\|_{\mathbb{H}_{X}}^{2}+(\tilde{f}, k)_{\mathbb{H}_{X}}
$$

whence

$$
\langle k, \tilde{\gamma}\rangle=(k, \tilde{f})_{\mathbb{H}_{X}} \geq 0 .
$$

Since $k \in \mathbb{H}_{X}^{+}$is arbitrary, then $\tilde{\gamma} \in M^{+}\left(\mathbb{T}_{1}\right)$ by assumption (P), i.e. we have (G1). (G2) follows from Proposition 2.6, whereas (G3) follows from the definition of $\tilde{f}$. 
2.2. Asymptotics. In this section we derive expansions for $\log P_{y f, u}$ as $y$ tends to infinity. We will need the following additional assumption.

(D) $\mathcal{R}$ is injective on $M\left(\mathbb{T}_{1}\right)$.

Remark 2.8. Condition (D) is equivalent to the distribution of $X$ having full support, i.e. the support of the distribution of $X$ coincides with $C_{0}\left(\mathbb{T} ; \mathbb{T}_{0}\right)$. Indeed, it is well known (see e.g. [29, Lemma 5.1]) that the support of distribution of $X$ is the closure of $\mathcal{R} M\left(\mathbb{T}_{1}\right)$. If the latter were not $C_{0}\left(\mathbb{T} ; \mathbb{T}_{0}\right)$, then by Hanh-Banach theorem there would exist non-zero $\gamma \in M\left(\mathbb{T}_{1}\right)$ such that $\langle f, \gamma\rangle=0$ for all $f \in \mathcal{R} M\left(\mathbb{T}_{1}\right)$. In particular, $\langle\mathcal{R} \gamma, \gamma\rangle=0$, which would contradict the injectivity. On the other hand, if $\mathcal{R} \gamma=0$ for some non-zero $\gamma$, then $\langle f, \gamma\rangle=0$ for all $f \in \mathcal{R} M\left(\mathbb{T}_{1}\right)$, hence, for all $f$ from the support of $X$, which then cannot be full.

We have chosen the injectivity assumption because we believe it is easier to verify than the full support property.

Now we state the assumptions on the boundary function $u$.

(U) There exists a sequence $\left(u_{n}, n \geq 1\right)$ of continuous functions such that

1) $u_{n}(t) \uparrow u(t), n \rightarrow+\infty$, for all $t \in \mathbb{T}_{1}$;

2) $P_{0, u, u_{n}}=\mathrm{P}\left(\forall t \in \mathbb{T} u_{n}(t) \leq X_{t} \leq u(t)\right)>0$ for all $n \geq 1$.

Remark 2.9. Under assumption (D), a sufficient condition for a lower semicontinuous $u: \mathbb{T} \rightarrow \mathbb{R}$ to satisfy $(\mathrm{U})$ is that $u(t)>0$ for all $t \in \mathbb{T}_{0}$. Indeed, in this case for any $u_{-} \in C(\mathbb{T})$ such that $u_{-}(t)<0$ for all $t \in \mathbb{T}_{0}$ and $u_{-}(t)<u(t)$ for all $t \in \mathbb{T}$, the set

$$
A_{u, u_{-}}=\left\{g \in C_{0}\left(\mathbb{T} ; \mathbb{T}_{0}\right) \mid \forall t \in \mathbb{T} \quad u_{-}(t)<g(t)<u(t)\right\}
$$

is non-empty and open in $C_{0}\left(\mathbb{T} ; \mathbb{T}_{0}\right)$. Therefore, since the support of distribution of $X$ is $C_{0}\left(\mathbb{T} ; \mathbb{T}_{0}\right)$, then we have

$$
P_{0, u, u_{-}}=\mathrm{P}\left(X \in A_{u, u_{-}}\right)>0 .
$$

Consequently, (U) holds for any sequence of continuous functions $u_{n} \in C(\mathbb{T})$ such that $u_{n}(t)<0, t \in T_{0}$, and $u_{n}(t) \uparrow u(t), n \rightarrow+\infty$, for any $t \in \mathbb{T}_{1}$.

We believe that (U) holds whenever $u(t) \geq 0$ for $t \in \mathbb{T}_{0}$ and $P_{0, u}>0$. However, the above argument fails, as the set $A_{u, u_{-}}$can be empty. Considering the set

$$
B_{u, u_{-}}:=\left\{g \in C_{0}\left(\mathbb{T} ; \mathbb{T}_{0}\right) \mid \forall t \in \mathbb{T}_{1} \quad u_{-}(t) \leq g(t) \leq u(t)\right\}
$$

will not help, as it is not open in general. One could consider a finer topology to overcome this problem, but then the dual space would be larger and perhaps not as tractable as $M\left(\mathbb{T}_{1}\right)$.

Theorem 2.10. Assume that $(D)$ holds, $f \in \mathbb{H}_{X}$ and let $u: \mathbb{T} \rightarrow \mathbb{R}$ be a lower semicontinuous function satisfying (U). If there exists $\tilde{f}=\mathcal{R} \tilde{\gamma} \in \mathbb{H}_{X}$ satisfying (G1)-(G3), then

$$
\begin{aligned}
\log P_{y f, u} & =-\frac{y^{2}}{2}\|\tilde{\gamma}\|_{\mathcal{H}_{X}}^{2}+y \Theta(\tilde{\gamma}, u)+o(y) \\
& =-\frac{y^{2}}{2}\|\tilde{f}\|_{\mathbb{H}_{X}}^{2}+y \Theta(\tilde{\gamma}, u)+o(y), \quad y \rightarrow+\infty .
\end{aligned}
$$

Remark 2.11. It follows from (13) that all $\tilde{\gamma} \in \mathcal{H}_{X}$ satisfying (G1)-(G3) must have equal norms. Therefore, since the set of such functions is convex, they all must coincide in $\mathcal{H}_{X}$ implying that such $\tilde{\gamma} \in \mathcal{H}_{X}$ is unique.

Proof. Denote the right-hand side of (13) by $r(y, \tilde{\gamma}, u)$. Since $P_{0, u}>0$, the inequality (6) yields

$$
\limsup _{y \rightarrow+\infty}\left(\log P_{y f, u}-r(y, \tilde{\gamma}, u)\right) \leq \limsup _{y \rightarrow+\infty} \log P_{y(f-\mathcal{R} \tilde{\gamma}), u} \leq 0
$$


so it remains to establish the lower bound.

Next, take the sequence $\left(u_{n}, n \geq 1\right)$ satisfying $(\mathrm{U})$. It is clear that one can choose positive integers $(k=k(y), y \geq 0)$ growing to $+\infty$ as $y \rightarrow+\infty$ sufficiently slowly so that $y^{-1} \log P_{0, u, u_{k}} \rightarrow 0, y \rightarrow+\infty$. Then for any $n \geq 1$, by (77) we get

$$
\liminf _{y \rightarrow+\infty} y^{-1}\left(\log P_{y f, u}-r\left(y, \tilde{\gamma}, u_{k}\right)\right) \geq \liminf _{y \rightarrow+\infty} y^{-1} \log P_{0, u, u_{k}}=0 .
$$

Consequently,

$$
\begin{gathered}
\liminf _{y \rightarrow+\infty} y^{-1}\left(\log P_{y f, u}-r(y, \tilde{\gamma}, u)\right) \\
\geq \liminf _{y \rightarrow+\infty} y^{-1}\left(\log P_{y f, u}-r\left(y, \tilde{\gamma}, u_{k}\right)\right)+\liminf _{y \rightarrow+\infty} y^{-1}\left(r\left(y, \tilde{\gamma}, u_{k}\right)-r(y, \tilde{\gamma}, u)\right) \\
\geq \liminf _{y \rightarrow+\infty}\left(\Theta\left(\tilde{\gamma}, u_{k}\right)-\Theta(\tilde{\gamma}, u)\right)=\liminf _{y \rightarrow+\infty} \int_{\mathbb{T}_{1}}\left(u_{k}(t)-u(t)\right) \tilde{\gamma}(d t) .
\end{gathered}
$$

Thanks to the dominated convergence $\lim _{y \rightarrow+\infty} \int_{\mathbb{T}_{1}}\left(u_{k}(t)-u(t)\right) \tilde{\gamma}(d t)=0$ implying

$$
\liminf _{y \rightarrow+\infty} y^{-1}\left(\log P_{y f, u}-r(y, \tilde{\gamma}, u)\right) \geq 0
$$

hence the proof is complete.

We are now ready to state the main result of this section.

Theorem 2.12. Let $(D)$ and $(P)$ hold, $f \in \mathbb{H}_{X}$ and let $u$ be a lower semicontinuous function satisfying (U). If further $\tilde{\gamma}$ is the projection of 0 to the set $C_{f}$, then (13) holds.

Proof. The statement follows from Proposition 2.7 and Theorem 2.10.

In general, it is difficult to identify $\tilde{\gamma}$. But there are cases where it is possible, e.g. if the drift is the covariance operator applied to a non-negative measure. Namely, the following result follows from Theorem 2.12 immediately.

Corollary 2.13. Assume that $(D)$ holds and $u$ is a lower semicontinuous function satisfying (U). Then for any $\gamma \in M^{+}\left(\mathbb{T}_{1}\right)$ and $f=\mathcal{R} \gamma$ the asymptotic expansion (13) holds with $\tilde{\gamma}=\gamma$.

2.3. Locally compact parameter space. Let now the continuous centered Gaussian process $X$ be indexed by a separable metric space $\mathbb{T}$, which we will assume here to be noncompact, but locally compact. We want to reduce our problem to its counterpart with compact (separable) parameter set. To this end, denote by $\overline{\mathbb{T}}=\mathbb{T} \cup\left\{t_{\infty}\right\}$ the one-point compactification of $\mathbb{T}$. We first show that $X$ can be multiplied by some positive function so that the product vanishes at infinity.

Lemma 2.14. There exists a continuous function $v: \mathbb{T} \rightarrow(0, \infty)$ such that $v(t) X_{t} \rightarrow 0$, $t \rightarrow t_{\infty}$ a.s.

Proof. Being a separable metric space, the space $\mathbb{T}$ is Lindelöf, so in view of local compactness there exists a countable family $\left\{\mathbb{T}_{n}, n \geq 1\right\}$ of compact sets such that $\mathbb{T}=\bigcup_{n \geq 1} \mathbb{T}_{n}$ and $\mathbb{T}_{n}$ is contained in $\mathbb{T}_{n+1}^{\circ}$, the interior of $\mathbb{T}_{n+1}$, for each $n \geq 1$ (see e.g. [16, Chapter XI, Theorem 7.2]).

Since $X$ is continuous and for each $n \geq 1, \mathbb{T}_{n}$ is compact, then clearly

$$
\mathrm{P}\left(\sup _{t \in \mathbb{T}_{n}}\left|X_{t}\right|<\infty\right)=1
$$

Therefore, there exists some $a_{n}>0$ such that $\mathrm{P}\left(\sup _{t \in \mathbb{T}_{n}}\left|X_{t}\right|>a_{n}\right)<2^{-n}$. Without loss of generality, we can assume that $a_{n}<a_{n+1}$ for each $n \geq 1$ and $a_{n} \rightarrow \infty, n \rightarrow+\infty$. 
For any $n \geq 1$, denote $b_{n}=a_{n+1}^{-2}$ and $D_{n}=\partial \mathbb{T}_{n}:=\mathbb{T}_{n} \backslash \mathbb{T}_{n}^{\circ}$. Since $\mathbb{T}_{n} \subset \mathbb{T}_{n+1}^{\circ}$, we have $\partial \mathbb{T}_{n} \cap \partial \mathbb{T}_{n-1}=\varnothing$. Then by Urysohn's lemma, there exists a continuous function $v_{n}: \mathbb{T}_{n+1} \backslash \mathbb{T}_{n}^{\circ} \rightarrow\left[b_{n+1}, b_{n}\right]$ such that $v_{n}(t)=b_{n}, t \in D_{n}, v_{n}(t)=b_{n+1}, t \in D_{n+1}$. Now set

$$
v(t)=a_{1} \mathbb{1}_{\mathbb{T}_{1}}(t)+\sum_{n=1}^{\infty} v_{n}(t) \mathbb{1}_{\mathbb{T}_{n+1} \backslash \mathbb{T}_{n}}(t), t \in \mathbb{T} .
$$

By construction, this is a continuous function with $\sup _{\mathbb{T} \backslash \mathbb{T}_{n}} v(t) \leq b_{n}, n \geq 1$.

On the other hand, by the Borel-Cantelli lemma, with probability 1 there exists $n_{0}(\omega)$ such that $\sup _{t \in \mathbb{T}_{n}}\left|X_{t}\right| \leq a_{n}, n \geq n_{0}(\omega)$. Therefore, for $n \geq n_{0}(\omega)$

$$
\sup _{t \in \mathbb{T}_{n+1} \backslash \mathbb{T}_{n}}\left|v(t) X_{t}\right| \leq \sup _{\mathbb{T} \backslash \mathbb{T}_{n}} v(t) \cdot \sup _{t \in \mathbb{T}_{n+1}}\left|X_{t}\right| \leq a_{n+1}^{-2} \cdot a_{n+1}=a_{n+1}^{-1} .
$$

Consequently, for all $n \geq n_{0}(\omega)$

$$
\sup _{t \in \mathbb{T} \backslash \mathbb{T}_{n}}\left|v(t) X_{t}\right| \leq a_{n+1}^{-1} \rightarrow 0
$$

as $n \rightarrow+\infty$ establishing the proof.

Remark 2.15. The above lemma is valid for any continuous process on $\mathbb{T}$, since the Gaussian distribution of $X$ is not used in the proof.

Now we are ready to state the main result about reduction to the case of compact parameter space.

Namely, set below

$$
\bar{X}_{t}=v(t) X_{t}, \quad \bar{f}(t)=v(t) f(t), \quad \bar{u}(t)=v(t) u(t)
$$

and putting

$$
\bar{X}_{t_{\infty}}=\bar{f}\left(t_{\infty}\right)=0, \quad \bar{u}\left(t_{\infty}\right)=\left(\liminf _{t \rightarrow t_{\infty}} v(t) u(t)\right) \wedge 0
$$

we have the following statement.

Theorem 2.16. The process $\bar{X}$ is a continuous centered Gaussian process on $\overline{\mathbb{T}}$ and for any $f \in \mathbb{H}_{X}$ and any lower semicontinuous $u: \mathbb{T} \rightarrow \mathbb{R}$, we have that $\bar{f} \in \mathbb{H}_{\bar{X}}$, $\bar{u}$ is lower semicontinuous and further

$$
\mathrm{P}\left(\forall t \in \mathbb{T} \quad X_{t}+f(t) \leq u(t)\right)=\mathrm{P}\left(\forall t \in \overline{\mathbb{T}} \quad \bar{X}_{t}+\bar{f}(t) \leq \bar{u}(t)\right) .
$$

Remark 2.17. It is important e.g. for asymptotic results like (3) that $\bar{X}, \bar{f}$, and $\bar{u}$ depend linearly (and in a rather simple way) on $X, f$ and $u$, respectively.

Proof. The process $\bar{X}$ is obviously centered Gaussian, and Lemma 2.14 immediately implies that $\bar{X}$ is continuous. The fact that $\bar{f} \in \mathbb{H}_{\bar{X}}$ is a consequence of the following well-known characterization of the Cameron-Martin space. Namely, it consists of functions $f$ such that the distribution of $X+f$ is absolutely continuous w.r.t. that of $X$. That said, for any $\bar{A} \subset C(\overline{\mathbb{T}})$ such that $\mathrm{P}(\bar{X} \in \bar{A})=0$, define $A=\left\{\left.h\right|_{\mathbb{T}} / v, h \in \bar{A}\right\}$ and write

$$
\mathrm{P}(X \in A)=\mathrm{P}(\bar{X} \in \bar{A})=0 .
$$

Hence, since $f \in \mathbb{H}_{X}$ we have

$$
\mathrm{P}(\bar{X}+\bar{f} \in \bar{A})=\mathrm{P}(X+f \in A)=0,
$$

whence we derive that $\bar{f} \in \mathbb{H}_{\bar{X}}$. Equation (14) is obtained similarly.

It remains to remark that $\bar{u}$ is lower semicontinuous by definition. (It is possible that $\bar{u}\left(t_{\infty}\right)=-\infty$, but in this case, and more generally in the case where $u\left(t_{\infty}\right)<0$ both probabilities in question are equal to zero.) 
2.4. Relation to the large deviation principle. The asymptotics (3) is also closely related to the large deviation principle. Since for process $X$ is a centered Gaussian element in the separable Banach space $C_{0}\left(\mathbb{T} ; \mathbb{T}_{0}\right)$, we can apply the general large deviation principle by Donsker and Varadhan [15] (see also [13, Section 3.4], [14, Theorem 4.5]): for any Borel set $A \subset C_{0}\left(\mathbb{T} ; \mathbb{T}_{0}\right)$,

$$
-\inf _{A^{\circ}} I(x) \leq \liminf _{\varepsilon \rightarrow 0+} \varepsilon^{2} \log \mathrm{P}(X \in A) \leq \limsup _{\varepsilon \rightarrow 0+} \varepsilon^{2} \log \mathrm{P}(X \in A) \leq-\inf _{\bar{A}} I(x),
$$

where $A^{\circ}$ and $\bar{A}$ are the interior and the closure of $A$, respectively. If we assume, as before, the injectivity of the covariance operator, the rate functional can be identified through the concept of Wiener quadruple (see [13, p. 88]): the Banach space $C_{0}\left(\mathbb{T} ; \mathbb{T}_{0}\right)$ together with the Hilbert space $\mathbb{H}_{X}$, the identity map $S: \mathbb{H}_{X} \rightarrow C_{0}\left(\mathbb{T} ; \mathbb{T}_{0}\right.$ ) (which is injective thanks to our assumption) and the distribution of $X$ forms a Wiener quadruple, so the rate functional is given by [13, Theorem 3.4.12]:

$$
I(x)= \begin{cases}\frac{1}{2}\|x\|_{\mathbb{H}_{X}}^{2}, & x \in \mathbb{H}_{X}, \\ \infty, & x \notin \mathbb{H}_{X} .\end{cases}
$$

To relate the large deviation extimates (15) to the boundary non-crossing probability $P_{y f, u}$, denote $\varepsilon=y^{-1}$,

$$
A_{f}=\{g: \mathbb{T} \rightarrow \mathbb{R} \mid \forall t \in \mathbb{T} g(t) \leq-f(t)\} .
$$

Then the boundary non-crossing probability can be written as $P_{y f, u}=\mathrm{P}\left(\varepsilon X \in A_{f-\varepsilon u}\right)$, however, (15) is not directly applicable since the target set $A_{f-\varepsilon u}$ depends on $\varepsilon$. To overcome this problem, one may fix some $\varepsilon_{0}>0$ and write

$$
\begin{gathered}
\limsup _{\varepsilon \rightarrow 0+} \varepsilon^{2} \log \mathrm{P}\left(\varepsilon X \in A_{f-\varepsilon u}\right) \leq \limsup _{\varepsilon \rightarrow 0+} \varepsilon^{2} \log \mathrm{P}\left(\varepsilon X \in A_{f-\varepsilon_{0} u^{+}}\right) \\
\leq-\inf _{\bar{A}_{f-\varepsilon_{0} u^{+}}} I(x)=-\inf _{A_{f-\varepsilon_{0} u^{+}}} I(x),
\end{gathered}
$$

where $u^{+}(t)=\max \{u(t), 0\}$. Then, letting $\varepsilon_{0} \rightarrow+0$, we get

$$
\limsup _{\varepsilon \rightarrow 0+} \varepsilon^{2} \log \mathrm{P}\left(\varepsilon X \in A_{f-\varepsilon u}\right) \leq-\inf _{A_{f}} I(x) .
$$

But it is clear that

$$
\inf _{\bar{A}_{f}} I(x)=\frac{1}{2} \inf \left\{\|g\|_{\mathbb{H}_{X}}^{2}: g \leq-f\right\}=\frac{1}{2} \inf \left\{\|h\|_{\mathbb{H}_{X}}^{2}: h \geq f\right\}=\frac{1}{2}\|\tilde{f}\|_{\mathbb{H}_{X}}^{2},
$$

where $\tilde{f}$ is, as before, the solution to the constrained minimization problem (10). As a result, going back to our notation,

$$
\lim _{y \rightarrow+\infty} y^{-2} \log P_{y f, u} \leq-\frac{1}{2}\|\tilde{f}\|_{\mathbb{H}_{X}}^{2} .
$$

However, in the case where $\mathbb{T}_{0} \neq \varnothing$, there is no clear way how to get a lower bound from (15): when $u$ is non-negative, the same approach gives

$$
\limsup _{\varepsilon \rightarrow 0+} \varepsilon^{2} \log \mathrm{P}\left(\varepsilon X \in A_{f-\varepsilon u}\right) \geq \limsup _{\varepsilon \rightarrow 0+} \varepsilon^{2} \log \mathrm{P}\left(\varepsilon X \in A_{f}\right) \geq-\inf _{A_{f}^{\circ}} I(x) .
$$

Since both $X$ and $f$ vanish on $\mathbb{T}_{0}, A_{f}$ has empty interior, so $\inf _{A_{f}^{\circ}} I(x)=\infty$; the lower estimate given by the large deviation principle sharp, as $\mathrm{P}\left(\varepsilon X \in A_{f}\right)=0$ in many cases, e.g., for Brownian motion on $[0, T]$. 
As it was mentioned in Introduction, there is another (simpler) way to derive a lower bound, leading to (2), which, combined with (16), yields

$$
\log P_{y f, u} \sim-\frac{y^{2}}{2}\|\tilde{f}\|_{\mathbb{H}_{X}}^{2}, \quad y \rightarrow+\infty .
$$

Unfortunately, this gives only the main term of asymptotic expansion (3). The next term of the asymptotics comes from the following heuristics: the target set $A_{f-\varepsilon u}$ is almost $A_{f}$ with slightly perturbed boundary. Therefore, denoting by $\Lambda(A)$ the "rate functional" corresponding to the set $A$, and assuming some smoothness, one might expect that for $\varepsilon \rightarrow 0+$,

$$
\Lambda\left(A_{f-\varepsilon u}\right) \approx \Lambda\left(A_{f}\right)-\varepsilon\left\langle u, \Lambda_{f}^{\prime}\left(A_{f}\right)\right\rangle=\frac{1}{2}\|\tilde{f}\|_{\mathbb{H}_{X}}^{2}-\varepsilon\left\langle u, \Lambda^{\prime}\left(A_{f}\right)\right\rangle,
$$

where $\Lambda_{f}^{\prime}$ is the derivative in some sense of $\Lambda\left(A_{f}\right)$ with respect to $f$, and this relation looks similar to (3). In certain situations, this heuristic argument may be given a precise meaning: see, for example, [10, Theorem 9.3.2]. However, in our case such argument would most likely fail. Indeed, if it were possible to validate, it would also work for negative "perturbations". However, if $\mathbb{T}_{0} \neq \varnothing$ and $u$ is positive, then $\mathrm{P}\left(X \in A_{f+\varepsilon u}\right)=0$, since $X$ and $f$ both vanish on $\mathbb{T}_{0}$, so (17) cannot provide correct logarithmic asymptotics.

On the other hand, the large deviation estimates may be used to derive the asymptotic behavior of the probabilities $\mathrm{P}\left(\varepsilon X \in A_{f-\delta u}\right)$ when $\varepsilon \rightarrow 0+$ and then $\delta \rightarrow 0+$, similarly to the results for random walks, established in [9], but such questions are beyond the scope of our article.

\section{Applications}

In this section we specialize the general results of Section 2 to several one-parameter processes. In all the examples, we skip the routine verification of assumptions $(\mathrm{P})$ and (D) while putting more emphasis on relevant details.

Throughout the section, $W=\left\{W_{t}, t \in \mathbb{R}\right\}$ is a standard Wiener process on $(\Omega, \mathcal{F}, \mathrm{P})$. By $A C([a, b])$ we will denote the set of absolutely continuous functions defined on $[a, b]$, and for $f \in A C([a, b]), f^{\prime}$ will denote its weak derivative.

3.1. Wiener process on $[0, b]$. Let $\mathbb{T}=[0, b]$ and $X=W$. Now $\mathbb{T}_{0}=\{0\}, \mathbb{T}_{1}=\mathbb{T} \backslash \mathbb{T}_{0}=$ $(0, b]$. The primary space is $C_{0}([0, b])=\{x \in C([0, b]): x(0)=0\}$ with dual $M((0, b])$, the space of finite signed measures on $(0, b]$. The covariance operator is given by

$$
\begin{aligned}
& \mathcal{R} \mu(t)=\int_{0}^{T} \min (t, s) \mu(d s)=\int_{0}^{T} \int_{0}^{t} \mathbb{1}_{[0, s]}(u) d u \mu(d s) \\
= & \int_{0}^{t} \int_{0}^{T} \mathbb{1}_{[0, s]}(u) \mu(d s) d u=\int_{0}^{t} \mu([u, b]) d u=\int_{0}^{t} J \mu(u) d u,
\end{aligned}
$$

where $J \mu(u)=\mu([u, b]), u \in[0, b), J \mu(T)=\mu(\{T\})$. Similarly, for $\mu, \nu \in M((0, b])$

$$
\begin{aligned}
& \langle\mathcal{R} \mu, \nu\rangle=\int_{0}^{b} \int_{0}^{b} \min (t, s) \mu(d s) \nu(d t)=\int_{0}^{b} \int_{0}^{b} \int_{0}^{b} \mathbb{1}_{[0, t]}(u) \mathbb{1}_{[0, s]}(u) d u \mu(d s) \nu(d t) \\
& =\int_{0}^{b} \int_{0}^{b} \mathbb{1}_{[0, s]}(u) \mu(d s) \int_{0}^{b} \mathbb{1}_{[0, b]}(u) \nu(d t) d u=\int_{0}^{b} J \mu(u) J \nu(u) d u=(J \mu, J \nu)_{L^{2}[0, b]} .
\end{aligned}
$$

Consequently, $J$ extends to an isomorphism between $\mathcal{H}_{X}$ and $L^{2}[0, b]$; the image is full since $J \mu$ can be arbitrary left-continuous bounded variation function. Therefore, in view of (18), the image of $\mathcal{H}_{X}$ under the covariance operator consists of functions of the form 
$\int_{0}^{t} h(u) d u$, where $h \in L^{2}[0, b]$, which is the well-known description of the Cameron-Martin space of $W$. It is worth mentioning that for $\mu \in M((0, b])$ and $f=\mathcal{R} \mu$ we have

$$
\begin{gathered}
\int_{0}^{t} f^{\prime}(t) d W_{t}=\int_{0}^{b} J \mu(t) d W_{t}=\int_{0}^{b} \mu([t, b]) d W_{t}=\int_{0}^{b} \int_{t}^{b} \mu(d s) d W_{t} \\
=\int_{0}^{b} \int_{0}^{s} d W_{t} \mu(d s)=\int_{0}^{b} W_{s} \mu(d s)=\langle W, \mu\rangle,
\end{gathered}
$$

so the Cameron-Martin density can be transformed to its more familiar form:

$$
\begin{gathered}
\mathcal{E}_{W}(\mu)=\exp \left\{\langle W, \mu\rangle-\frac{1}{2}\|\mu\|_{\mathcal{H}_{X}}^{2}\right\}=\exp \left\{\int_{0}^{t} f^{\prime}(t) d W_{t}-\frac{1}{2}\|J \mu\|_{L^{2}[0, b]}^{2}\right\} \\
=\exp \left\{\int_{0}^{t} f^{\prime}(t) d W_{t}-\frac{1}{2}\left\|f^{\prime}\right\|_{L^{2}[0, b]}^{2}\right\} .
\end{gathered}
$$

Further, the image of a non-negative finite measure on $(0, b]$ is an absolutely continuous function $f$ with $f(0)=0$ and with a non-increasing non-negative derivative. Equivalently, this is a concave non-decreasing function with $f(0)=0$. Therefore, in order to identify the function $\tilde{f}$ from Theorem 2.10, which corresponds to the drift $f=\mathcal{R} \gamma$, we need to find a concave non-decreasing function $\tilde{f} \geq f$ such that $\tilde{\gamma}=\mathcal{R}^{-1} \tilde{f}$ satisfies (G2). The latter is equivalent to

$$
(J \gamma-J \tilde{\gamma}, J \tilde{\gamma})_{L^{2}[0, b]} \geq 0
$$

which, in view of (18), reads

$$
\left(f^{\prime}-\tilde{f}^{\prime}, \tilde{f}^{\prime}\right)_{L^{2}[0, b]} \geq 0
$$

Thanks to Theorem A.1, this property (even with equality) is satisfied by the least nondecreasing concave majorant of $f$, which also is a solution to the minimization problem (10). This is not surprising, as we recover the well-known results for the Wiener process (see e.g. 4]), which we summarize below. Note also that, by definition of $J \gamma$, we should define $\tilde{f}^{\prime}$ to be left-continuous on $(0, b]$ and continuous at zero, so we should take the left derivative for $t \in(0, b]$ and the right derivative at 0 .

Theorem 3.1. Let $u:[0, b] \rightarrow \mathbb{R}$ be lower semicontinuous, $f \in A C([0, b])$ be such that $f(0)=0$ and $f^{\prime} \in L^{2}[0, b], \tilde{f}$ be the least non-decreasing concave majorant of $f$, and $\tilde{f}_{-}^{\prime}(t)$ be its left derivative (right derivative for $t=0$ ).

1. The probability $P_{f, u}=\mathrm{P}\left(\forall t \in[0, b] W_{t}+f(t) \leq u(t)\right)$ admits the upper bound

$$
P_{f, u} \leq P_{f-\tilde{f}, u} \exp \left\{-\frac{1}{2}\left\|\tilde{f}_{-}^{\prime}\right\|_{L^{2}[0, b]}^{2}+\int_{0}^{b} u(t) d\left(-\tilde{f}_{-}^{\prime}(t)\right)\right\} .
$$

2. For any $u_{-} \in C([0, b])$ such that $u_{-}(t)<u(t)$ for all $t \in[0, b]$ and

$$
P_{0, u, u_{-}}:=\mathrm{P}\left(\forall t \in[0, b] \quad u_{-}(t) \leq W_{t} \leq u(t)\right)>0,
$$

the probability $P_{f, u}$ admits the lower bound

$$
P_{f, u} \geq P_{0, u, u_{-}} \exp \left\{-\frac{1}{2}\left\|\tilde{f}_{-}^{\prime}\right\|_{L^{2}[0, b]}^{2}+\int_{0}^{b} u_{-}(t) d\left(-\tilde{f}_{-}^{\prime}(t)\right)\right\} .
$$

3. If $u(0)>0$, then the following asymptotics holds:

$$
\log P_{y f, u}=-\frac{y^{2}}{2}\left\|\tilde{f}_{-}^{\prime}\right\|_{L^{2}[0, b]}^{2}+y \int_{0}^{b} u(t) d\left(-\tilde{f}_{-}^{\prime}(t)\right)+o(y), \quad y \rightarrow+\infty .
$$

3.2. Wiener process on $[a, b]$. Let again $X=W$, but $\mathbb{T}=[a, b]$ with $a<b$, $a b \neq 0$. There are two different cases depending on whether $0 \in[a, b]$ or not. 
3.2.1. $0 \notin[a, b]$. In this case $\mathbb{T}_{0}=\varnothing, \mathbb{T}_{1}=[a, b]$. The primary space is $C([a, b])$ with dual $M([a, b])$, the space of finite signed measures on $[a, b]$. Without loss of generality, we can assume $a>0$.

Similarly to (18), the covariance operator is given by

$$
\begin{aligned}
\mathcal{R} \mu(t) & =\int_{a}^{b} \int_{0}^{t} \mathbb{1}_{[0, s]}(u) d u \mu(d s)=\int_{0}^{t} \int_{a}^{b} \mathbb{1}_{[0, s]}(u) \mu(d s) d u \\
& =\int_{0}^{t} \mu([u \vee a, b]) d u=\int_{0}^{t} J_{a} \mu(u) d u, t \in[a, b],
\end{aligned}
$$

where $J_{a} \mu(u)=\mu([u \vee a, b])$; also for $\mu, \nu \in M([a, b])$ we have

$$
\langle\mathcal{R} \mu, \nu\rangle=\int_{a}^{b} J_{a} \mu(u) J_{a} \nu(u) d u=\left(J_{a} \mu, J_{a} \nu\right)_{L^{2}[0, b]} .
$$

As above, the operator $J_{a}$ extends to an isomorphism between $\mathcal{H}_{X}$ and some subspace of $L^{2}[0, b]$. The image is now not full, since, for each $\mu \in M([a, b]), J_{a} \mu$ is constant on $[0, a]$; in fact, it is easy to see that $J_{a} \mathcal{H}_{X}$ consists of square integrable functions which are constant on $[0, a]$. Then, by (19), the image of $\mathcal{H}_{X}$ under the covariance operator consists of absolutely continuous functions on $[a, b]$ with square integrable derivative. The image of $M^{+}([a, b])$ is a bit trickier. As in the previous example, by (19), it contains concave non-decreasing functions, but not all of them. In fact, it is easy to see from (19) that we must have $f_{+}^{\prime}(a)=\mu((a, b]) \leq \mu([a, b])=f(a) / a \geq 0$; also every concave nondecreasing function with such property belongs to the image. Now the function $\tilde{f}$ from Theorem 2.10 corresponding to the drift $f=\mathcal{R} \gamma$ is a concave non-decreasing function such that $\tilde{f}_{+}^{\prime}(a) \geq \tilde{f}_{a}(a) \geq 0$ and $\tilde{\gamma}=\mathcal{R}^{-1} \tilde{f}$ satisfies (G2). As in the previous example, it is possible to identify this function. Namely, thanks to the isomorphism property of $J_{a}$, we can rewrite (G2) as

$$
\left(J_{a} \gamma-J_{a} \tilde{\gamma}, J_{a} \tilde{\gamma}\right)_{L^{2}[0, b]} \geq 0
$$

We have $J_{a} \gamma(t)=f^{\prime}(t)$ for $t \in[a, b]$, and $J_{a} g$ is constant on $[0, a]$ with $\int_{0}^{a} J_{a} \gamma(t)=f(a)$. So, if we extend $f$ to $[0, a]$ linearly, i.e. $f(t)=t f(a) / a, t \in[0, a]$, then we have $J_{a} \gamma(t)=$ $f^{\prime}(t)$ for $t \in[0, b]$. Then, for the least concave non-decreasing majorant $\tilde{f}$ of $f$, we have by Theorem A.1 that

$$
\left(f^{\prime}-\tilde{f}^{\prime}, \tilde{f}^{\prime}\right)_{L^{2}[0, b]}=0 .
$$

Moreover, $\tilde{f}^{\prime}$ is clearly constant on $[0, a]$, so we have $\tilde{f}_{-}^{\prime}=J_{a} \tilde{\gamma}$, where $\tilde{\gamma}$ is the non-negative measure on $[a, b]$ given by $\tilde{\gamma}([t, b])=-\tilde{f}_{-}^{\prime}(t), t \in[a, b]$, and (20) follows. Hence we arrive at the following result.

Theorem 3.2. Let $u:[a, b] \rightarrow \mathbb{R}$ be lower semicontinuous and $f \in A C([a, b])$ be such that $f^{\prime} \in L^{2}[a, b]$. Define $f(t)=t f(a) / a$ for $t \in[0, a]$, let $\tilde{f}:[0, b] \rightarrow \mathbb{R}$ be the least non-decreasing concave majorant of $f$ on $[0, b]$ and $\tilde{f}_{-}^{\prime}$ be its left derivative.

1. The probability $P_{f, u}=\mathrm{P}\left(\forall t \in[a, b] W_{t}+f(t) \leq u(t)\right)$ admits the upper bound

$$
P_{f, u} \leq P_{f-\tilde{f}, u} \exp \left\{-\frac{1}{2}\left\|\tilde{f}_{-}^{\prime}\right\|_{L^{2}[0, b]}^{2}+\int_{a}^{b} u(t) d\left(-\tilde{f}_{-}^{\prime}(t)\right)\right\} .
$$

2. For any $u_{-} \in C([a, b])$ such that $u_{-}(t)<u(t)$ for all $t \in[a, b]$ and

$$
P_{0, u, u_{-}}:=\mathrm{P}\left(\forall t \in[a, b] \quad u_{-}(t) \leq W_{t} \leq u(t)\right)>0,
$$

the probability $P_{f, u}$ admits the lower bound

$$
P_{f, u} \geq P_{0, u, u_{-}} \exp \left\{-\frac{1}{2}\left\|\tilde{f}_{-}^{\prime}\right\|_{L^{2}[0, b]}^{2}+\int_{a}^{b} u_{-}(t) d\left(-\tilde{f}_{-}^{\prime}(t)\right)\right\} .
$$


3. The following asymptotics holds:

$$
\log P_{y f, u}=-\frac{y^{2}}{2}\left\|\tilde{f}_{-}^{\prime}\right\|_{L^{2}[0, b]}^{2}+y \int_{a}^{b} u(t) d\left(-\tilde{f}_{-}^{\prime}(t)\right)+o(y), \quad y \rightarrow+\infty .
$$

Remark 3.3. Actually, this example can be compared with the previous one. Namely, we can informally write

$$
P_{f, u}=\mathrm{P}\left(\forall t \in[a, b] \quad W_{t}+f(t) \leq u(t)\right) \approx \mathrm{P}\left(\forall t \in[0, b] \quad W_{t}+f(t) \leq u(t)\right),
$$

with some $f$, which has large negative values on $[0, a)$. Of course, the latter is impossible if $f(a)>0$, since $f$ must be continuous, but with suitable approximation argument it is possible to derive Theorem 3.2 from Theorem 3.1 .

3.2.2. $0 \in[a, b]$. Now $a<0, b>0, \mathbb{T}_{0}=\{0\}, \mathbb{T}_{1}=[a, 0) \cup(0, b]$. The primary space is $C_{0}([a, b] ;\{0\})=\{f \in C([a, b]): f(0)=0\}$ with dual $M([a, 0) \cup(0, b])$.

The covariance function is equal to $R(t, s)=t \wedge s$, for $t, s>0,-(t \vee s)$ for $t, s<0$, and 0 if $t s \leq 0$. Then for $t \geq 0$ the covariance operator is

$$
\mathcal{R} \mu(t)=\int_{a}^{b} R(t, s) \mu(d s)=\int_{0}^{b} \int_{0}^{t} \mathbb{1}_{[0, s]}(u) d u \mu(d s)=\int_{0}^{t} \mu([u, b]) d u=\int_{0}^{t} J \mu(u) d u,
$$

where $J \mu(u)=\mu([u, b]), u \in[0, b]$. Similarly, for $t \in[a, 0)$

$$
\mathcal{R} \mu(t)=\int_{t}^{0} \mu([a, u]) d u
$$

where $J \mu(u)=\mu([a, u]), u \in[a, 0)$, and

$$
\langle\mathcal{R} \mu, \nu\rangle=(J \mu, J \nu)_{L^{2}[a, b]} \cdot
$$

Consequently, the operator $J$ extends to an isomorphism between $\mathcal{H}_{X}$ and $L^{2}[a, b]$ and

$$
\mathbb{H}_{X}=\left\{f \in A C([a, b]): f(0)=0, f^{\prime} \in L^{2}[a, b]\right\} .
$$

As a result, we get a similar situation as for $[0, b]$. The difference is that now the function $\tilde{f}$ is non-decreasing and concave on $[0, b]$ but non-increasing and concave on $[a, 0]$, so it can be "glued" together from the least non-decreasing concave majorant of $f$ on $[0, b]$ and the least non-increasing concave majorant on $[a, 0]$. The bounds and the asymptotic behavior we obtain are similar to the previous statements, so we skip the formulation. The important fact we should mention is that the values of $W$ on $[a, 0]$ and $[0, b]$ are independent, so we can write

$$
\mathrm{P}(W+f \leq u \text { on }[a, b])=\mathrm{P}(W+f \leq u \text { on }[a, 0]) \cdot \mathrm{P}(W+f \leq u \text { on }[0, b])
$$

and apply Theorem 3.1. The results will agree with those obtained by direct application of the general theory, since

$$
\left\|\tilde{f}^{\prime}\right\|_{L^{2}[a, b]}^{2}=\left\|\tilde{f}^{\prime}\right\|_{L^{2}[a, 0]}^{2}+\left\|\tilde{f}^{\prime}\right\|_{L^{2}[0, b]}^{2}
$$

and

$$
\int_{a}^{b} u(t) d\left(-\tilde{f}^{\prime}(t)\right)=\int_{a}^{0} u(t) d\left(-\tilde{f}^{\prime}(t)\right)+\int_{0}^{b} u(t) d\left(-\tilde{f}^{\prime}(t)\right)
$$


3.3. Brownian bridge. For convenience in this example we work with $\mathbb{T}=[0,1]$. Let $X_{t}=B_{t}^{0}:=W_{t}-t W_{1}, t \in[0,1]$, be a Brownian bridge, which is a centered Gaussian process with covariance function $R(t, s)=\min (t, s)-t s$. The primary space is now

$$
C_{0,0}([0,1])=\{x \in C([0,1]): x(0)=x(1)=0\},
$$

with the dual space $M((0,1))$. Further, similarly to (18) the covariance operator is given by

$$
\begin{gathered}
\mathcal{R} \mu(t)=\int_{0}^{1}(\min (t, s)-t s) \mu(d s)=\int_{0}^{t} \mu([s, 1)) d s-t \int_{0}^{1} \int_{0}^{s} d u \mu(d s)= \\
\int_{0}^{t} \mu([s, 1)) d s-t \int_{0}^{1} \mu([u, 1)) d u=\int_{0}^{t}\left(\mu((s, 1))-\int_{0}^{1} \mu([u, 1)) d u\right) d s=\int_{0}^{t} J_{0} \mu(s) d s,
\end{gathered}
$$

where $J_{0} \mu(s)=\mu([s, 1))-\int_{0}^{1} \mu([u, 1)) d u$. Using simple transformations, we obtain

$$
\langle\mathcal{R} \mu, \nu\rangle=\int_{0}^{b} J_{0} \mu(u) J_{0} \nu(u) d u=\left(J_{0} \mu, J_{0} \nu\right)_{L^{2}[0,1]} .
$$

Consequently, $J_{0}$ extends to an isometry between $\mathcal{H}_{X}$ and the completion of image of $J_{0}$ in $L^{2}[0,1]$, which easily seen to be

$$
L_{0}^{2}[0,1]:=\left\{f \in L^{2}[0,1]: \int_{0}^{1} f(t) d t=0\right\} .
$$

Hence, in view of (21), the Cameron-Martin space $\mathbb{H}_{X}=\mathcal{R} \mathcal{H}_{X}$ consists of absolutely continuous functions having square integrable derivative and vanishing at 0 and 1 , which agrees with the well known description of this RKHS, see e.g. [23, Example 4.9]. Similarly to the previous example, the drift $\tilde{f}$ from Theorem 2.9 should satisfy $\tilde{f} \geq f$ and

$$
\left(f^{\prime}-\tilde{f}^{\prime}, \tilde{f}^{\prime}\right)_{L^{2}[0,1]} \geq 0 \text {. }
$$

By Lemma A.2, this is true for the least concave majorant of $f$, which is also a solution to (10). So again we reproduce the known results for Brownian bridge, see [3, [5, 7].

Theorem 3.4. Let $u:[0,1] \rightarrow \mathbb{R}$ be lower semicontinuous, $f \in A C([0,1])$ be such that $f(0)=f(1)=0, f^{\prime} \in L^{2}[0,1], \tilde{f}$ be the least concave majorant of $f$, and $\tilde{f}_{-}^{\prime}$ be its left derivative (right derivative at 0 ).

1. The probability $P_{f, u}=\mathrm{P}\left(\forall t \in[0,1] B_{t}^{0}+f(t) \leq u(t)\right)$ admits the upper bound

$$
P_{f, u} \leq P_{f-\tilde{f}, u} \exp \left\{-\frac{1}{2}\left\|\tilde{f}_{-}^{\prime}\right\|_{L^{2}[0,1]}^{2}+\int_{0}^{1} u(t) d\left(-\tilde{f}_{-}^{\prime}(t)\right)\right\}
$$

2. For any $u_{-} \in C([0,1])$ such that $u_{-}(t)<u(t)$ for all $t \in[0,1]$ and

$$
P_{0, u, u_{-}}:=\mathrm{P}\left(\forall t \in[0,1] \quad u_{-}(t) \leq B_{t}^{0} \leq u(t)\right)>0,
$$

the probability $P_{f, u}$ admits the lower bound

$$
P_{f, u} \geq P_{0, u, u_{-}} \exp \left\{-\frac{1}{2}\left\|\tilde{f}_{-}^{\prime}\right\|_{L^{2}[0,1]}^{2}+\int_{0}^{1} u_{-}(t) d\left(-\tilde{f}_{-}^{\prime}(t)\right)\right\} .
$$

3. If $u(0), u(1)>0$, then we have

$$
\log P_{y f, u} \sim-\frac{y^{2}}{2}\left\|\tilde{f}_{-}^{\prime}\right\|_{L^{2}[0,1]}^{2}+y \int_{0}^{1} u(t) d\left(-\tilde{f}_{-}^{\prime}(t)\right)+o(y), \quad y \rightarrow+\infty .
$$


3.4. Brownian motion on $[0,+\infty)$. Let $X=W, \mathbb{T}=[0,+\infty)$. Now $\mathbb{T}$ is locally compact, so we should use the ideas of Subsection 2.3. But first we transform the parameter space conveniently, setting

$$
Y_{t}=W_{t /(1-t)}, t \in[0,1) .
$$

Now we should multiply $Y$ by some positive function $v \in C([0,1))$ so that $v(t) Y_{t} \rightarrow 0$, $t \rightarrow 1-$. It is not hard to see that $v(t)=1-t$ works. As a result, we can write

$$
\mathrm{P}\left(\forall t \geq 0 W_{t}+f(t) \leq u(t)\right)=\mathrm{P}\left(\forall t \in[0,1] Z_{t}+\bar{f}(t) \leq \bar{u}(t)\right),
$$

where

$$
Z_{t}=(1-t) W_{t /(1-t)}, \quad \bar{f}(t)=(1-t) f(t /(1-t)), \quad \bar{u}(t)=(1-t) u(t /(1-t)) .
$$

It appears that the process $Z$ is a Brownian bridge on $[0,1]$, so we reduce the problem to the previous example; the solution $\tilde{f}$ to the constrained optimization problem is now a least non-decreasing concave majorant, as in Example 3.1 (see also [6, Lemma 5.1]).

3.5. Volterra process. Consider $X_{t}=\int_{0}^{t} K(t, s) d W_{s}, t \in[0, T]$, where the Volterra kernel $K$ is such that $\sup _{t \in[0, T]} \int_{0}^{t} K(t, s)^{2} d s<\infty$ and $X$ has continuous sample paths. In this case for any finite signed measure $\mu$ on $[0, T]$

$$
\begin{gathered}
\mathcal{R} \mu(t)=\int_{0}^{T} R(t, s) \mu(d s)=\int_{0}^{T} \int_{0}^{t \wedge s} K(t, u) K(s, u) d u \mu(d s) \\
=\int_{0}^{t} K(t, u) \int_{u}^{T} K(s, u) \mu(d s) d u .
\end{gathered}
$$

Consequently, the covariance operator admits the following decomposition $\mathcal{R}=\mathcal{K K}^{*}$, where

$$
\mathcal{K} f(t)=\int_{0}^{t} K(t, s) f(s) d s, \quad \mathcal{K}^{*} \mu(s)=\int_{s}^{T} K(t, s) \mu(d t) .
$$

Moreover, we have

$$
\begin{aligned}
\langle\mu, \nu\rangle= & \int_{0}^{T} \int_{0}^{T} R(t, s) \mu(d s) \nu(d s)=\int_{0}^{T} \int_{0}^{T} \int_{0}^{t \wedge s} K(t, u) K(s, u) d u \mu(d s) \nu(d s) \\
& =\int_{0}^{T} \int_{u}^{T} K(t, u) \mu(d t) \int_{u}^{T} K(s, u) \nu(d s) d u=\left(\mathcal{K}^{*} \mu, \mathcal{K}^{*} \nu\right)_{L^{2}[0, T]}^{2} .
\end{aligned}
$$

As a result, $\mathcal{H}_{X}$ can be identified with a preimage of $L^{2}[0, T]$ under $\mathcal{K}^{*}$, and $\mathbb{H}_{X}$, with the image of $L^{2}[0, T]$ under $\mathcal{K}$. Despite the seemingly clear, as in the previous examples, description of the Cameron-Martin space, it is in general hard to identify the solution of the minimization problem (10). (See, for example, the article [21], which considers the boundary non-crossing probabilities for fractional Brownian motion, in particular Theorem 3.1 and Corollary 3.2 therein.) Of course, there is a viable case contained in Corollary 2.13; for any $\gamma \in M([0, T])$ and $f=\mathcal{R} \gamma$, the asymptotic expansion (13) holds, however, the Volterra structure does not really help here.

3.6. Brownian sheet. Let $X$ be a Brownian sheet, i.e. a centered Gaussian process indexed by $\mathbb{T}=[0, T]^{2}$ and having the covariance function

$$
R\left(\left(t_{1}, t_{2}\right),\left(s_{1}, s_{2}\right)\right)=\min \left(t_{1}, s_{1}\right) \cdot \min \left(t_{2}, s_{2}\right),\left(t_{1}, t_{2}\right),\left(s_{1}, s_{2}\right) \in \mathbb{T} .
$$

Now $\mathbb{T}_{0}=(\{0\} \times[0, T]) \cup([0, T] \times\{0\}), \mathbb{T}_{1}=\mathbb{T} \backslash \mathbb{T}_{0}=(0, T]^{2}$. The primary space is $C_{0}\left([0, T]^{2} ; \mathbb{T}_{0}\right)=\{x \in C([0, T]): \forall t \in[0, T] x(0, t)=x(t, 0)=0\}$ with dual $M\left((0, T]^{2}\right)$, 
the space of finite signed measures on $(0, T]^{2}$. Similarly to Example 3.1, the covariance operator is

$$
\begin{gathered}
\mathcal{R} \mu\left(t_{1}, t_{2}\right)=\int_{0}^{T} \int_{0}^{T} \min \left(t_{1}, s_{1}\right) \min \left(t_{2}, s_{2}\right) \mu\left(d s_{1}, d s_{2}\right) \\
=\int_{0}^{t_{1}} \int_{0}^{t_{2}} \mu\left(\left[u_{1}, T\right] \times\left[u_{2}, T\right]\right) d u_{2} d u_{1}=\int_{0}^{t_{1}} \int_{0}^{t_{2}} J_{2} \mu\left(u_{1}, u_{2}\right) d u_{2} d u_{1},
\end{gathered}
$$

where $J_{2} \mu\left(u_{1}, u_{2}\right)=\mu\left(\left[u_{1}, T\right] \times\left[u_{2}, T\right]\right)$, and

$$
\langle\mathcal{R} \mu, \nu\rangle=\left(J_{2} \mu, J_{2} \nu\right)_{L^{2}\left([0, T]^{2}\right)},
$$

so $J_{2}$ extends to an isomorphism between $\mathcal{H}_{X}$ and $L^{2}\left([0, T]^{2}\right)$. Therefore, $\mathbb{H}_{X}$ consists of functions of the form $\int_{0}^{t_{1}} \int_{0}^{t_{2}} h\left(u_{1}, u_{2}\right) d u_{1} d u_{2}$, where $h \in L^{2}\left([0, T]^{2}\right)$, so again we get the well-known description of the Cameron-Martin space of Brownian sheet.

We do not know the solution to the optimization problem (10) in general, only in two particular case. The first case is where $f$ is itself the solution, then we have an ad hoc version of Corollary 2.13 .

Theorem 3.5. Let $X$ be a Brownian sheet, $u:[0, T]^{2} \rightarrow \mathbb{R}$ be a lower semicontinuous function such that $u(0, t)>0$ and $u(t, 0)>0$ for all $t \in[0, T], \gamma$ be a finite non-negative measure on $(0, T]^{2}$, and

$$
f\left(t_{1}, t_{2}\right)=\int_{0}^{t_{1}} \int_{0}^{t_{2}} J_{2} \gamma\left(u_{1}, u_{2}\right) d u_{2} d u_{1}=\int_{0}^{t_{1}} \int_{0}^{t_{2}} \gamma\left(\left[u_{1}, T\right] \times\left[u_{2}, T\right]\right) d u_{2} d u_{1} .
$$

Then, the following asymptotics holds:

$$
\begin{gathered}
\log \mathrm{P}\left(\forall t_{1}, t_{2} \in[0, T] \quad X_{t_{1}, t_{2}}+y f\left(t_{1}, t_{2}\right) \leq u\left(t_{1}, t_{2}\right)\right) \\
=-\frac{y^{2}}{2}\left\|J_{2} \gamma\right\|_{L^{2}\left([0, T]^{2}\right)}^{2}+y \int_{0}^{T} \int_{0}^{T} u\left(t_{1}, t_{2}\right) \gamma\left(d t_{1}, d t_{2}\right)+o(y), \quad y \rightarrow+\infty .
\end{gathered}
$$

The second case is $f\left(t_{1}, t_{2}\right)=f_{1}\left(t_{1}\right) \cdot f_{2}\left(t_{2}\right)$ with non-negative $f_{1}, f_{2}$ belonging to the RKHS of Wiener space, i.e., $f_{i}=\int_{0}^{t} h_{i}(s) d s$ with $h_{i} \in L^{2}[0, T], i=1,2$. In this case, the solution to the optimization problem is $\tilde{f}\left(t_{1}, t_{2}\right)=\tilde{f}_{1}\left(t_{1}\right) \cdot \tilde{f}_{2}\left(t_{2}\right)$, where $\tilde{f}_{i}$ is the smallest non-decreasing concave majorant of $f_{i}, i=1,2$. Indeed, $\tilde{f} \geq f$ and, thanks to Lemma A.1

$$
\int_{0}^{T} \tilde{f}_{i}^{\prime}(s)\left(f_{i}^{\prime}(s)-\tilde{f}_{i}^{\prime}(s)\right) d s=0, \quad i=1,2,
$$

hence

$$
\begin{gathered}
\int_{0}^{T} \int_{0}^{T} f_{1}^{\prime}\left(s_{1}\right) f_{2}^{\prime}\left(s_{2}\right) \tilde{f}_{1}^{\prime}\left(s_{1}\right) \tilde{f}_{2}^{\prime}\left(s_{2}\right) d s_{1} d s_{2} \\
=\int_{0}^{T} f_{1}^{\prime}\left(s_{1}\right) \tilde{f}_{1}^{\prime}\left(s_{1}\right) d s_{1} \int_{0}^{T} f_{2}^{\prime}\left(s_{2}\right) \tilde{f}_{2}^{\prime}\left(s_{2}\right) d s_{2} \\
=\int_{0}^{T} \tilde{f}_{1}^{\prime}\left(s_{1}\right)^{2} d s_{1} \int_{0}^{T} \tilde{f}_{2}^{\prime}\left(s_{2}\right)^{2} d s_{2}=\int_{0}^{T} \int_{0}^{T} \tilde{f}_{1}^{\prime}\left(s_{1}\right)^{2} \tilde{f}_{2}^{\prime}\left(s_{2}\right)^{2} d s_{1} d s_{2},
\end{gathered}
$$

equivalently,

$$
\int_{0}^{T} \int_{0}^{T}\left(f_{1}^{\prime}\left(s_{1}\right) f_{2}^{\prime}\left(s_{2}\right)-\tilde{f}_{1}^{\prime}\left(s_{1}\right) \tilde{f}_{2}^{\prime}\left(s_{2}\right)\right) \tilde{f}_{1}^{\prime}\left(s_{1}\right) \tilde{f}_{2}^{\prime}\left(s_{2}\right) d s_{1} d s_{2}=0
$$


As in Example 3.1, assuming that $f=\mathcal{R} \gamma, \tilde{f}=\mathcal{R} \tilde{\gamma}$, the last equality is equivalent to (G2). Thus, noting that

$$
\|\tilde{\gamma}\|_{\mathbb{H}_{X}}=\|\tilde{f}\|_{L^{2}\left([0, T]^{2}\right)}=\left\|\tilde{f}_{1}\right\|_{L^{2}[0, T]} \cdot\left\|\tilde{f}_{1}\right\|_{L^{2}[0, T]},
$$

we arrive at the following statement.

Theorem 3.6. Let $X$ be a Brownian sheet, $u:[0, T]^{2} \rightarrow \mathbb{R}$ be a lower semicontinuous such that $u(0, t)>0$ and $u(t, 0)>0$ for all $t \in[0, T]$. Let also $f_{1}, f_{2} \in A C([0, T])$ be nonnegative functions such that $f_{i}(0)=0$ and $f_{i}^{\prime} \in L^{2}[0, T], i=1,2$, and $\tilde{f}_{1}, \tilde{f}_{2}$ be their least concave non-decreasing majorants. Then, the following asymptotics holds as $y \rightarrow+\infty$ :

$$
\begin{gathered}
\log \mathrm{P}\left(\forall t_{1}, t_{2} \in[0, T] \quad X_{t_{1}, t_{2}}+y f_{1}\left(t_{1}\right) f_{2}\left(t_{2}\right) \leq u\left(t_{1}, t_{2}\right)\right) \\
=-\frac{y^{2}}{2}\left\|\tilde{f}_{1}\right\|_{L^{2}[0, T]}^{2} \cdot\left\|\tilde{f}_{2}\right\|_{L^{2}[0, T]}^{2}+y \int_{0}^{T} \int_{0}^{T} u\left(t_{1}, t_{2}\right) d \tilde{f}_{1}^{\prime}\left(t_{1}\right) d \tilde{f}_{2}^{\prime}\left(t_{2}\right)+o(y) .
\end{gathered}
$$

\section{Appendix A. Auxiliary statements}

The following lemma summarizes properties of the least non-decreasing concave majorant. They are probably well known, but here we write them for completeness.

Lemma A.1. For a function $f \in A C([0, T])$ with $f(0)=0$, its least non-decreasing concave majorant $\tilde{f}$ exists and is also absolutely continuous with $\tilde{f}(0)=0$. Moreover, if $f^{\prime} \in L^{2}[0, T]$, then $\tilde{f}^{\prime} \in L^{2}[0, T]$ and

$$
\int_{0}^{T}\left(f^{\prime}(s)-\tilde{f}^{\prime}(s)\right) \tilde{f}^{\prime}(s) d s=0, \quad \tilde{f}=\underset{g \in \mathbb{H}_{W}, g \geq f}{\operatorname{argmin}}\left\|g^{\prime}\right\|_{L^{2}[0, T]},
$$

where

$$
\mathbb{H}_{W}=\left\{g:[0, T] \rightarrow \mathbb{R}: f(t)=\int_{0}^{t} h(s) d s, \quad h \in L^{2}[0, T]\right\}
$$

is the RKHS of a standard Wiener process $W$.

Proof. Let $t_{0}=\operatorname{argmax}_{[0, T]} f$. The least non-decreasing concave majorant is non-decreasing on $\left[0, t_{0}\right]$ and constant on $\left[t_{0}, T\right]$ with $\tilde{f}\left(t_{0}\right)=f\left(t_{0}\right)$, so it is enough to prove the statement on $\left[0, t_{0}\right]$ given $t_{0}>0$. To simplify the notation, we will assume $t_{0}=T$.

Since $\tilde{f}$ is non-decreasing on $[0, T]$ and exceeds $f$, it is not less than the least nondecreasing majorant $\hat{f}(t)=\max _{s \in[0, t]} f(s)$ of $f$. Further, for all $x<y, \hat{f}(y)-\hat{f}(x)$ does not exceed the variation of $f$ on $x, y$, which is equal to $\int_{x}^{y}\left|f^{\prime}(s)\right| d s$. Therefore, $\hat{f}$ is absolutely continuous with $\left|\hat{f}^{\prime}(t) \leq\right| f^{\prime}(t) \mid$ a.e., in particular, $\left|\hat{f}^{\prime}\right|$ is square integrable. Consequently, it is enough to prove the statement for a non-decreasing $f$ (equivalently, for non-negative $f^{\prime}$ ).

Let $h$ denote the monotone rearrangement of $f^{\prime}($ i.e. $h(t)=\sup \{x: \lambda(\{s \in[0, T]$ : $\left.\left.\left.\left.f^{\prime}(s) \geq x\right\}\right) \leq t\right\}, t \in[0, T]\right)$. It is well-known that $\int_{0}^{T} h(t)^{2} d t=\int_{0}^{T} f^{\prime}(t)^{2} d t$ and for all $t \in[0, T]$

$$
\int_{0}^{t} h(s) d s \geq \int_{0}^{t} f^{\prime}(s) d s=f(t)
$$

Since $h$ is non-increasing, $g(t):=\int_{0}^{t} h(s) d s$ is a non-decreasing concave majorant of $f$. Moreover, $g$ is continuous with $g(0)=0$ and $g(T)=f(T)$. Therefore, $\tilde{f}$, being the least non-decreasing concave majorant, lies between $f$ and $g$, so it is also continuous at 0 and $T$ with $\tilde{f}(0)=0, \tilde{f}(T)=f(T)$. Since $\tilde{f}$ is non-decreasing, it can only have jump discontinuities, which, however, would condradict concavity, so it is continuous. 
Now let $Z=\{t \in[0, T]: \tilde{f}(t)=f(t)\}$. Since $f$ and $\tilde{f}$ are continuous, this set is closed with $\{0, T\} \subset Z$. Its complement is an open set, so it is a union of disjoint open intervals, say, $\bigcup_{n \geq 1}\left(a_{n}, b_{n}\right)$. Now for any $n \geq 1, \tilde{f}$ is affine on $\left[a_{n}, b_{n}\right]$ with $\tilde{f}\left(a_{n}\right)=f\left(a_{n}\right)$, $\tilde{f}\left(b_{n}\right)=f\left(b_{n}\right)$. Therefore, denoting for any $n \geq 1 \quad \bar{f}_{n}=\frac{f\left(b_{n}\right)-f\left(a_{n}\right)}{b_{n}-a_{n}}$, we have

$$
\int_{a_{n}}^{b_{n}}\left(f^{\prime}(s)-\tilde{f}^{\prime}(s)\right) \tilde{f}^{\prime}(s) d s=\bar{f}_{n} \int_{a_{n}}^{b_{n}}\left(f^{\prime}(s)-\bar{f}_{n}\right) d s=0,
$$

whence

$$
\begin{gathered}
\int_{0}^{T}\left(f^{\prime}(s)-\tilde{f}^{\prime}(s)\right) \tilde{f}^{\prime}(s) d s=\int_{Z}\left(f^{\prime}(s)-\tilde{f}^{\prime}(s)\right) \tilde{f}^{\prime}(s) d s \\
+\sum_{n \geq 1} \int_{a_{n}}^{b_{n}}\left(f^{\prime}(s)-\tilde{f}^{\prime}(s)\right) \tilde{f}^{\prime}(s) d s=0 .
\end{gathered}
$$

Since for each $n \geq 1, \int_{a_{n}}^{b_{n}} \tilde{f}^{\prime}(s)^{2} d s \leq \int_{a_{n}}^{b_{n}} f^{\prime}(s)^{2} d s$ by Jensen's inequality, then $\tilde{f}^{\prime} \in L^{2}[0, T]$ follows.

Further, since $\left\|\tilde{f}^{\prime}\right\|_{L^{2}[0, T]} \leq\left\|f^{\prime}\right\|_{L^{2}[0, T]}$, the minimiser of $\|g\|_{L^{2}[0, T]}$ for all $g \geq f, g \in H_{W}$ belongs to the set $A_{g}:=\left\{g \geq \tilde{f}, g \in H_{W}\right\}$ and $g$ is concave, non-decreasing. Consequently, it belongs also to the set $A_{g}^{*}=\left\{g \in A_{g}, g(T)=\tilde{f}(T)\right\}$. For any $g \in A_{g}^{*}$ we have

$$
\int_{0}^{T}\left(g^{\prime}(s)-\tilde{f}^{\prime}(s)\right) \tilde{f}^{\prime}(s) d s=\int_{0}^{T}(g(s)-\tilde{f}(s)) d\left(-\tilde{f}^{\prime}(s)\right) \geq 0,
$$

hence

$$
\left\|g^{\prime}\right\|_{L^{2}[0, T]}^{2}=\left\|g^{\prime}-\tilde{f}^{\prime}\right\|_{L^{2}[0, T]}^{2}+\left\|\tilde{f}^{\prime}\right\|_{L^{2}[0, T]}^{2}+\int_{0}^{T}\left(g^{\prime}(s)-\tilde{f}^{\prime}(s)\right) \tilde{f}^{\prime}(s) d s \geq\left\|\tilde{f}^{\prime}\right\|_{L^{2}[0, T]}^{2}
$$

and therefore the minimizer is unique and equals $\tilde{f}$ establishing the claim.

The following statement for Brownian bridge is proved similarly and therefore we omit its proof.

Lemma A.2. For an absolutely continuous function $f:[0, T] \rightarrow \mathbb{R}$, with $f(0)=f(T)=0$, its least concave majorant $\tilde{f}$ is also absolutely continuous with $\tilde{f}(0)=\tilde{f}(T)=0$. Moreover, if $f^{\prime} \in L^{2}[0, T]$, then $\tilde{f}^{\prime} \in L^{2}[0, T]$ and

$$
\int_{0}^{T} \tilde{f}^{\prime}(s)\left(\tilde{f}^{\prime}(s)-f^{\prime}(s)\right) d s=0, \quad \tilde{f}=\underset{g \in \mathbb{H}_{B^{0}}, g \geq f}{\operatorname{argmin}}\left\|g^{\prime}\right\|_{L^{2}[0, T]},
$$

where

$$
\mathbb{H}_{B^{0}}=\left\{g:[0, T] \rightarrow \mathbb{R}: f(t)=\int_{0}^{t} h(s) d s, h \in L^{2}[0, T], \int_{0}^{T} h(t) d t=0\right\}
$$

is the RKHS of a Brownian bridge $B^{0}$.

\section{ACKNOWLEDGEMENTS}

The authors thank anonymous referees for their careful reading of the manuscript and valuable remarks which helped to improve the article. Financial support from SNSF Grant 200021-175752/1 is kindly acknowledged. 


\section{REFERENCES}

[1] Frank Aurzada and Steffen Dereich. Universality of the asymptotics of the one-sided exit problem for integrated processes. Ann. Inst. Henri Poincaré Probab. Stat., 49(1), 2013.

[2] Frank Aurzada and Thomas Simon. Persistence probabilities and exponents. In Lévy matters. V, volume 2149 of Lecture Notes in Math., pages 183-224. Springer, Cham, 2015.

[3] Wolfgang Bischoff and Enkelejd Hashorva. A lower bound for boundary crossing probabilities of Brownian bridge/motion with trend. Statist. Probab. Lett., 74(3):265-271, 2005.

[4] Wolfgang Bischoff, Enkelejd Hashorva, and Jürg Hüsler. An asymptotic result for non crossing probabilities of Brownian motion with trend. Comm. Statist. Theory Methods, 36(13-16):2821-2828, 2007.

[5] Wolfgang Bischoff, Enkelejd Hashorva, Jürg Hüsler, and Frank Miller. Exact asymptotics for boundary crossings of the Brownian bridge with trend with application to the Kolmogorov test. Ann. Inst. Statist. Math., 55(4):849-864, 2003.

[6] Wolfgang Bischoff, Enkelejd Hashorva, Jürg Hüsler, and Frank Miller. Analysis of a change-point regression problem in quality control by partial sums processes and Kolmogorov type tests. Metrika, 62(1):85-98, 2005.

[7] Wolfgang Bischoff, Frank Miller, Enkelejd Hashorva, and Jürg Hüsler. Asymptotics of a boundary crossing probability of a Brownian bridge with general trend. Methodol. Comput. Appl. Probab., $5(3): 271-287,2003$.

[8] Wolfgang Bischoff and Wayan Somayasa. The limit of the partial sums process of spatial least squares residuals. J. Multivariate Anal., 100(10):2167-2177, 2009.

[9] A. A. Borovkov. Boundary value problems for random walks and large deviations in function spaces. Teor. Verojatnost. i Primenen., 12:635-654, 1967.

[10] Alexandr A. Borovkov. Probability theory. Universitext. Springer, London, 2013.

[11] Konstantin Borovkov and Alex Novikov. Explicit bounds for approximation rates of boundary crossing probabilities for the Wiener process. J. Appl. Probab., 42(1):82-92, 2005.

[12] Ward Cheney and Allen A. Goldstein. Proximity maps for convex sets. Proc. Amer. Math. Soc., 10:448-450, 1959.

[13] Jean-Dominique Deuschel and Daniel W. Stroock. Large deviations, volume 137 of Pure and Applied Mathematics. Academic Press, Inc., Boston, MA, 1989.

[14] Roland Dobrushin, Groeneboom Piet, and Bernard Pierre. Lectures on Probability Theory and Statistics. Ecole d'Eté de Probabilités de Saint-Flour XXIV - 1994, volume 1648 of Lecture Notes in Mathematics. Springer, Berlin, 1996.

[15] M. D. Donsker and S. R. S. Varadhan. Asymptotic evaluation of certain Markov process expectations for large time III. Comm. Pure Appl. Math., 29:389-461, 1976.

[16] James Dugundji. Topology. Allyn and Bacon, Inc., Boston, Mass., 1966.

[17] Robert E. Edwards. Functional analysis. Dover Publications, Inc., New York, 1995. Theory and applications, Corrected reprint of the 1965 original.

[18] Enkelejd Hashorva. Exact asymptotics for boundary crossing probabilities of Brownian motion with piecewise linear trend. Electron. Comm. Probab., 10:207-217, 2005.

[19] Enkelejd Hashorva. Boundary non-crossings of Brownian pillow. J. Theoret. Probab., 23(1):193-208, 2010.

[20] Enkelejd Hashorva and Yuliya Mishura. Boundary noncrossings of additive Wiener fields. Lith. Math. J., 54(3):277-289, 2014.

[21] Enkelejd Hashorva, Yuliya Mishura, and Oleg Seleznjev. Boundary non-crossing probabilities for fractional Brownian motion with trend. Stochastics, 87(6):946-965, 2015.

[22] Wenbo V. Li and James Kuelbs. Some shift inequalities for Gaussian measures. In High dimensional probability (Oberwolfach, 1996), volume 43 of Progr. Probab., pages 233-243. Birkhäuser, Basel, 1998.

[23] Mikhail A. Lifshits. Lectures on Gaussian processes. Springer Briefs in Mathematics. Springer, Heildelberg, 2012.

[24] Shaun McKinlay and Konstantin Borovkov. On approximation rates for boundary crossing probabilities for the multivariate Brownian motion process. Commun. Stoch. Anal., 9(2):267-282, 2015.

[25] Muhammad Aslam Noor. Some iterative algorithms for extended general variational inequalities. Albanian J. Math., 2(4):265-275, 2008.

[26] Alex Novikov, Volf Frishling, and Nino Kordzakhia. Time-dependent barrier options and boundary crossing probabilities. Georgian Math. J., 10(2):325-334, 2003. Dedicated to the memory of Professor Revaz Chitashvili. 
[27] Yuqing Pan and Konstantin A. Borovkov. The exact asymptotics of the large deviation probabilities in the multivariate boundary crossing problem. Adv. in Appl. Probab., 51(3):835-864, 2019.

[28] Klaus Pötzelberger and Liqun Wang. Boundary crossing probability for Brownian motion. J. Appl. Probab., 38(1):152-164, 2001.

[29] Aad van der Vaart and Harry van Zanten. Reproducing kernel Hilbert spaces of Gaussian priors. In Pushing the limits of contemporary statistics: contributions in honor of Jayanta K. Ghosh, volume 3 of Inst. Math. Stat. (IMS) Collect., pages 200-222. Inst. Math. Statist., Beachwood, OH, 2008. 